\title{
Augmented reality 3D navigation system for percutaneous CT-guided pulmonary ground-glass opacity biopsies: a comparison with the standard CT-guided technique
}

\author{
Eliodoro Faiella ${ }^{1}$, Laura Messina ${ }^{1}$, Gennaro Castiello ${ }^{1}$, Caterina Bernetti ${ }^{1}$, Giuseppina Pacella ${ }^{1}$, \\ Carlo Altomare ${ }^{1}$, Flavio Andresciani ${ }^{1}$, Marina Sarli ${ }^{1}$, Filippo Longo ${ }^{2}$, Pierfilippo Crucitti ${ }^{2}$, \\ Bruno Beomonte Zobel ${ }^{1}$, Rosario Francesco Grasso ${ }^{1}$
}

\begin{abstract}
${ }^{1}$ Diagnostic and Interventional Radiology, Departmental Falculty of Medicine and Surgery, Università Campus Bio-medico di Roma, Rome, Italy; ${ }^{2}$ Department of Thoracic Surgery, Departmental Falculty of Medicine and Surgery, Università Campus Bio-medico di Roma, Rome, Italy

Contributions: (I) Conception and design: E Faiella, L Messina, RF Grasso; (II) Administrative support: G Castiello, C Bernetti, G Pacella, C Altomare, F Andresciani, M Sarli, F Longo, P Crucitti, B Beomonte Zobel; (III) Provision of study materials or patients: All authors; (IV) Collection and assembly of data: All authors; (V) Data analysis and interpretation: E Faiella, L Messina, RF Grasso; (VI) Manuscript writing: All authors; (VII) Final approval of manuscript: All authors.

Correspondence to: Eliodoro Faiella. Diagnostic and Interventional Radiology, Departmental Falculty of Medicine and Surgery, Università Campus Bio-medico di Roma, 00128 Rome, Italy. Email: faiellaeliodoro@gmail.com.
\end{abstract}

Background: Augmented reality navigation system for percutaneous computed tomography (CT)-guided pulmonary biopsies has recently been introduced. There are no studies in literature about its use for ground glass lesions biopsies. The aim of this study is to evaluate the effectiveness of an augmented reality infrared navigation system performance on CT-guided percutaneous lung ground glass opacity (GGO) biopsy compared to a standard CT-guided technique.

Methods: A total of 80 patients with lung GGO who underwent to a percutaneous CT-guided lung biopsy with an augmented reality infrared navigation system were retrospectively enrolled in the study. Comparison was performed with a group of 80 patients who underwent to lung biopsy with the standard CT-guided technique. Evaluation of maximum lesion diameter (MLD), distance between lesion and pleural surface (DPS), distance travelled by the needle (DTP), procedural time, validity of histological sample, procedural complications and the radiation dose to the patient's chest were recorded for each patient of both groups. In addition, each group was divided into two subgroups based on lesion size, according to a cut-off of $1.5 \mathrm{~cm}$ $(<1.5 \mathrm{~cm} ; \geq 1.5 \mathrm{~cm})$.

Results: Augmented reality navigation system showed a significant reduction in procedural time, radiation dose administrated to patients and complications rate compared to a standard CT-guided technique. Technical success was achieved in the $100 \%$ of cases in both groups, but the diagnostical success was higher in the group where patients underwent to lung biopsies with the use of navigation system. We also found that using an augmented reality navigation system increases the diagnostical success rate for lesion $<1.5 \mathrm{~cm}$. MLD, DPS and DTP did not differ significantly between the two groups of patients.

Conclusions: The use of an augmented reality navigation system for percutaneous CT-guided pulmonary GGO biopsies has demonstrated a lower incidence of post-procedural complications, a significantly reduction of the radiation dose administered to patients and a higher diagnostical success rate.

Keywords: Ground glass opacity (GGO); augmented reality navigation system; percutaneous lung biopsy (PLB)

Submitted Aug 06, 2021. Accepted for publication Dec 15, 2021.

doi: $10.21037 /$ jtd-21-1285

View this article at: https://dx.doi.org/10.21037/jtd-21-1285 


\section{Introduction}

Pulmonary ground glass opacity (GGO) is a non-specific sign, defined as an area of increased attenuation in the lung on computed tomography (CT) that does not obscure underlying bronchial structures or pulmonary vessels. It includes both benign and malignant lesions. Recently, the incidence of GGO lesions has increased because of the widespread use of low-dose CT screening for lung cancer detection. There is no conclusive classification of lung GGO, but according to some authors, it could be distinguished in two types, pure and mixed; while others classify it in pure, heterogeneous, and part-solid (1). Table 1 shows the guidelines for management of incidentally detected lung GGO in adults according to Fleischner Society, 2017. Less than $1 \%$ of all patients has a malignant transformation of a pure GGO $\leq 5 \mathrm{~mm}$ in size and this is a strong evidence for a conservative treatment to the most of these typically non-invasive lesions (2). However, several studies have shown that persistent GGO on CT should be suspected with a high risk of malignancy, in fact the incidence of lung cancer is higher in persistent pure ground-glass nodules (GGNs) $\geq 10 \mathrm{~mm}$ in diameter (about $78 \%)(3,4)$. So, biopsy should be considered in case of persistent GGOs.

Percutaneous lung biopsy (PLB) has been shown to have high sensitivity, specificity and accuracy for characterization of pulmonary nodules, particularly if malignancy is suspected. Augmented reality navigation system for percutaneous CTguided pulmonary biopsies has recently been introduced (5). Such systems afford optical, electromagnetic or hybrid tracking of the devices during procedures, allowing a more accurate targeting of small or heterogeneous lung lesions, in order to have an adequate and representative tissue sample, necessary for the identification of specific biomarkers and activated pathways (6-8). Relatively new navigation models are based on an infrared optical localization system with a 3D-visualization to identify the position and direction of the interventional instruments in relation to both patient and lesion. In a previous experience, we have already shown that this navigation system (intra-operative robotic interventional system "SIRIO”, MASMEC BIOMED Spa, Modugno, Italy) reduces procedural time, pneumothorax rate and gives a better histological result in lesions $<20 \mathrm{~mm}$ compared to a standard CT-guided technique (9). We validated a low dose protocol to reduce significative patients' radiation exposure (10). In addition, we demonstrated that navigation system is a safe and effective CT-guided navigation system for PLB, independently on lesion size and location in a larger sample of patients with solid lung nodules (11).

There are no studies in literature about the use of an augmented reality navigation system for ground glass lesions biopsies.

The aim of this study is to evaluate the effectiveness of an augmented reality infrared navigation system performance on CT-guided percutaneous lung ground-glass opacity biopsy compared to a standard CT-guided technique.

We present the following article in accordance with the STROBE reporting checklist (available at https://jtd. amegroups.com/article/view/10.21037/jtd-21-1285/rc).

\section{Methods}

We retrospectively included in the study a total of 80 patients with lung GGO suspected of malignancy who had percutaneous CT-guided biopsy using navigation system at the Department of Interventional Radiology of our institution from June 2017 to September 2019. For the control group, we retrospectively reviewed the cases of 80 patients with GGO who underwent to CT-guided lung biopsy without using SIRIO. The study was conducted in accordance with the Declaration of Helsinki (as revised in 2013). This study was approved by the Institutional Review Board of the University Hospital of Campus Bio-Medico in Rome (01/17 OSS ComEt CBM) and we obtained a written informed consent from each enrolled patient both to undergo percutaneous CT-guided lung biopsy and to be included in the study.

Inclusion criteria for GGO PLB were considered: GGO with part solid $>5 \mathrm{~mm}$, GGO increasing in size during imaging follow-up and persistent GGO in patient with family history or with past lung tumour surgery.

Exclusion criteria were: anticoagulant medication not stopped at least 5 days before the procedure, personal history of coagulopathy, ECOG/WHO Performance Status Score $>3$, the absence of patient's compliance, age $<18$ years and pregnancy.

Both inclusion and exclusion criteria were the same for the two groups.

The navigation system works with infrared light and it includes an infrared localization apparatus to detect position and orientation of the needle, a processing unit for $3 \mathrm{D}$ reconstruction of the lung region, a disposable sterile kit with both patient and needle sensors and a tracking system for monitoring patient's movements and breathing. The infrared light is reflected by some spheres located on the 
Table 1 Fleischner Society 2017 guidelines for management of incidentally detected pulmonary GGO in adults

\begin{tabular}{llll}
\hline GGN type & Size $<6 \mathrm{~mm}$ & Size $\geq 6 \mathrm{~mm}$ & Comments \\
\hline Single & No routine follow-up & $\begin{array}{l}\text { CT at 6-12 months to confirm } \\
\text { persistence, then CT every 2 years until } \\
5 \text { years }\end{array}$ & $\begin{array}{l}\text { In certain suspicious nodules, 6 mm, consider } \\
\text { follow-up at 2 and 4 years. If solid component(s) } \\
\text { or growth develops, consider resection }\end{array}$ \\
Multiple & $\begin{array}{l}\text { CT at 3-6 months. If stable, } \\
\text { consider CT at 2 and 4 years }\end{array}$ & $\begin{array}{l}\text { CT at 3-6 months. Subsequent } \\
\text { management based on the most } \\
\text { suspicious nodule(s) }\end{array}$ & $\begin{array}{l}\text { Multiple, 6 mm pure GGNs are usually benign, but } \\
\text { consider follow-up in selected patients at high } \\
\text { risk at 2 and 4 years }\end{array}$ \\
\hline
\end{tabular}

GGO, ground glass opacity; GGN, ground-glass nodules; CT, computed tomography.

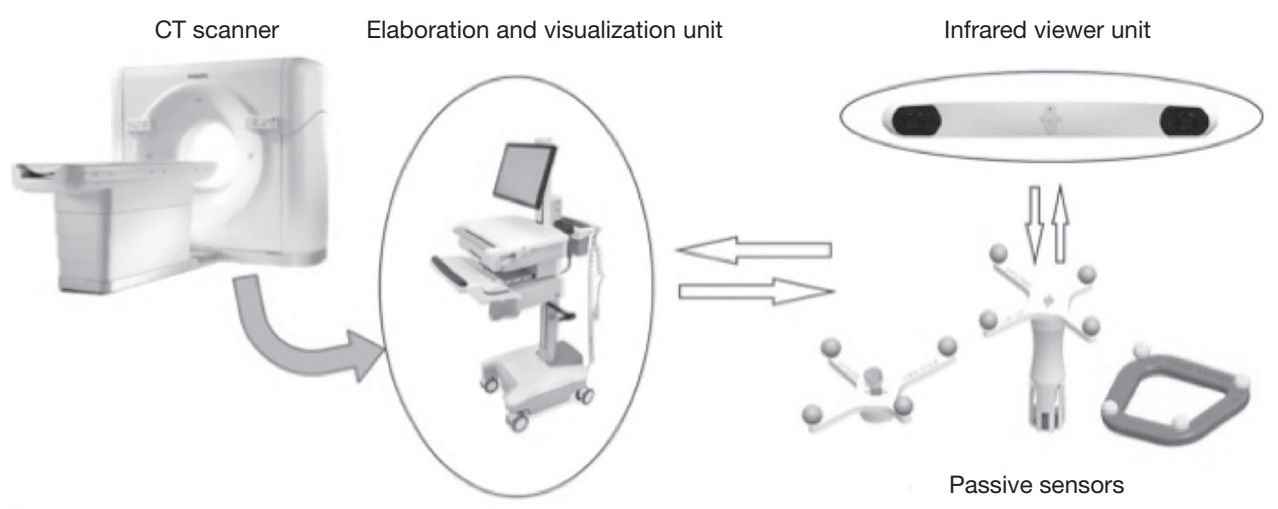

Figure 1 Navigation system elements. CT scanner, elaboration and visualization SIRIO unit and the sensors system (composed by needle sensor, patient's sensor and photo sensor positioned in the room roof) are shown. CT, computed tomography.

needle handle and on the patient's chest and it is detected by a photo sensor positioned in the room roof. The navigation system manages DICOM CT-images providing the position of the passive spheres placed on the patient's chest: a 3D patient's chest virtual model is created. Needle advancement is done with the help of the feedback from the needle path shown on the elaborating unit, considering patient's breathing, and it do not require a CT re-imaging for each progression. The navigation system is supported by a compensation feature for respiratory movement artifacts that allows to perform the procedure independently of patient free breathing.

Practically, we want to illustrate all the steps required to perform a lung biopsy with SIRIO. A chest CT is necessary, and it must also include the sensor located on the patient's chest. The DICOM imagines obtained are sent from the CT console to the SIRIO elaboration unit by cable. When the $3 \mathrm{D}$ imagine is available on the SIRIO visualization unit, needle calibration is required, and it is done by putting the needle sensor on the patient's sensor. The needle is now recognized, and it can be moved using the photo sensor positioned in the room roof.
Navigation system elements are summarized in Figure 1.

We used a 64-MDCT scanner (Somatom Sensation, Siemens, Forchheim, Germany) with a low-dose protocol. The following parameters were utilized: $100 \mathrm{kV}$, $35 \mathrm{mAs}, 64 \times 0.6-\mathrm{mm}$ detector configuration, table speed $0.81 \mathrm{~mm} /$ rotation, pitch $1.4,0.33$-s gantry rotation, slice thickness $2.3 \mathrm{~mm}$, reconstruction interval $1 \mathrm{~mm}$. In all cases, a soft tissue kernel was performed. For each patient, CT imaging was focused to the target area identified by reviewing the previous medical and radiological records available. An 18-gauge core-needle biopsy with a 17-gauge coaxial technique in blocked breathing was performed for each patient (examples in Figures 2,3) to obtain more than one sample using a single pleural path, reducing pneumothorax and seeding complications. The coaxial technique consists in positioning a guide needle in correspondence of the target lesion. Then a biopsy needle is introduced through the coaxial guide needle to reach the lesion and to obtain the sample. In non-coaxial biopsy the same procedure is performed only with the biopsy needle without the use of a coaxial guide needle. So, the needle is moved many times to obtain more samples of tissue, 

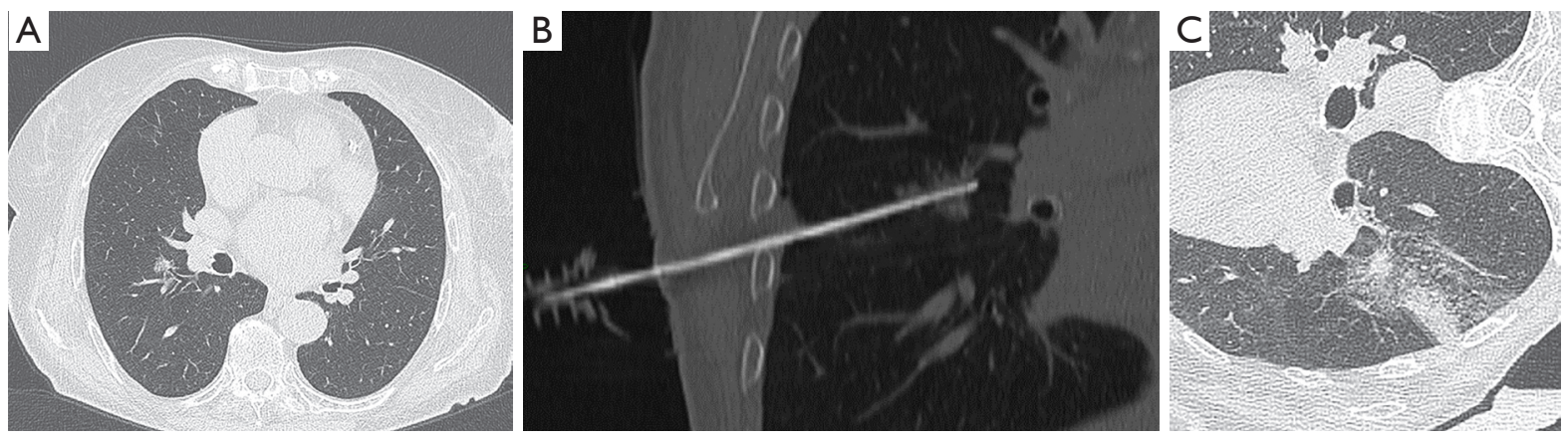

Figure 2 Low dose CT images showing GGO in upper segment of LRL about $13 \times 7 \mathrm{~mm}$ in diameter next to the hilum and adherent to the pleural fissure in 84 years old female patient under double antiplatelet therapy. Axial (A) and reformatted axial plane (B) along needle path images showing the correct needle placement into GGO. Final post-PLB CT control (C) showing a self-limited small focus of haemorrhage without PTX. Histological analysis reveals adenocarcinoma. CT, computed tomography; GGO, ground glass opacity; LRL, lower right lobe; PLB, percutaneous lung biopsy; PTX, pneumothorax.
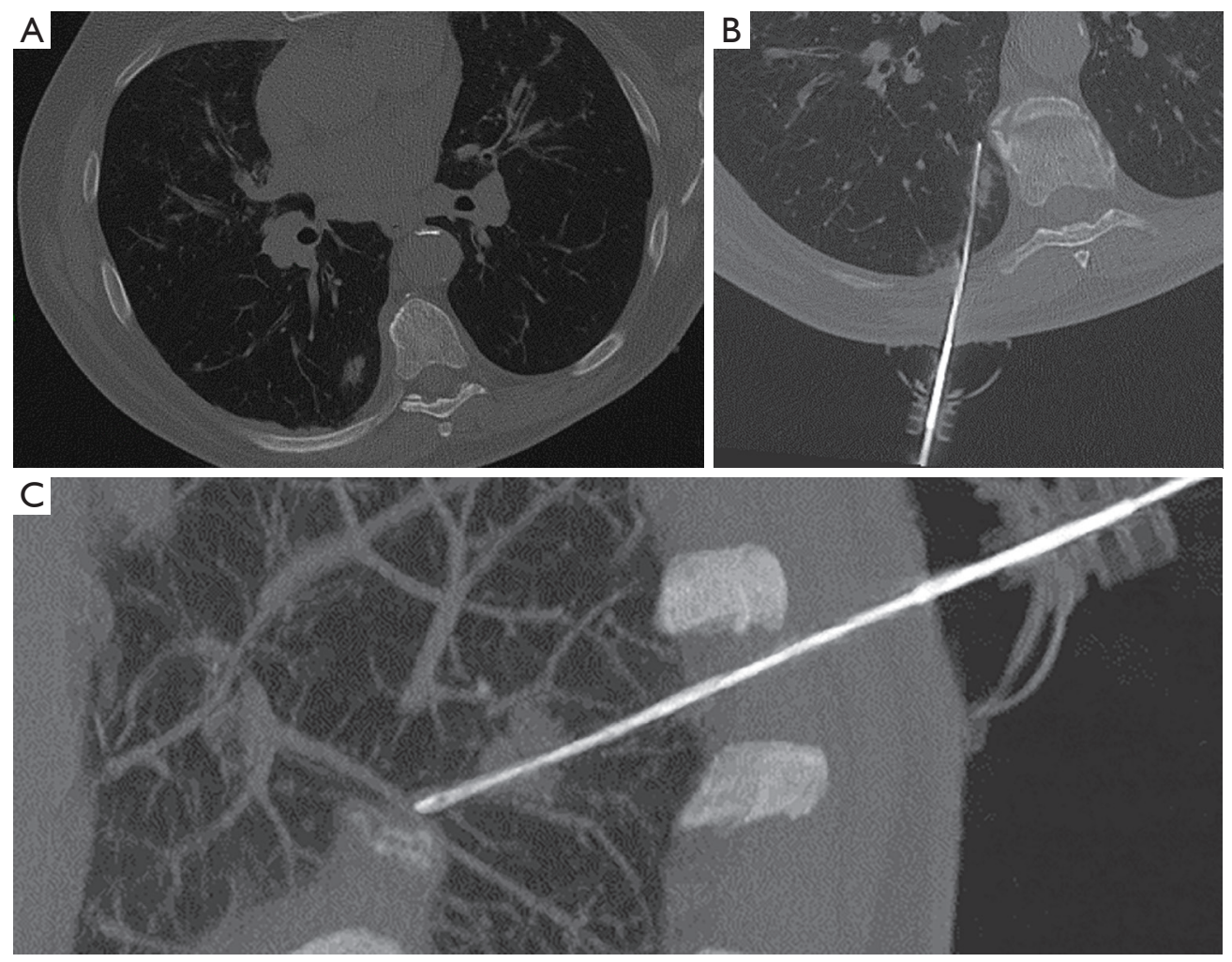

Figure 3 Low dose CT image (A) showing GGO in upper segment of LRL about $14 \times 9 \mathrm{~mm}$ in 72 years old man patient with a history of chronic lymphocytic leukemia and prostatic adenocarcinoma. Axial MPR (B) and sagittal MIP reconstruction (C) images along needle track. Histological analysis was adenocarcinoma. CT, computed tomography; GGO, ground glass opacity; LRL, lower right lobe; MPR, multiplanar reconstruction; MIP, maximum intensity projection. 
Table 2 Descriptive statistical analysis

\begin{tabular}{|c|c|c|c|c|c|c|c|c|}
\hline Group & $\begin{array}{l}\text { MLD } \\
(\mathrm{mm})\end{array}$ & $\begin{array}{l}\text { DPS } \\
(\mathrm{mm})\end{array}$ & $\begin{array}{l}\text { DTP } \\
(\mathrm{mm})\end{array}$ & $\begin{array}{l}\text { PT } \\
(\min )\end{array}$ & $\begin{array}{l}\text { Effective radiation } \\
\text { dose (mSv) }\end{array}$ & $\begin{array}{c}\text { Major complications } \\
\text { rate (\%) }\end{array}$ & $\begin{array}{c}\text { Minor complications } \\
\text { rate }(\%)\end{array}$ & $\begin{array}{c}\text { Diagnostical success } \\
\text { rate (\%) }\end{array}$ \\
\hline Trial & 16 & 13.5 & 71 & 15 & 5.5 & $2.5(2 / 80)$ & $30(24 / 80)$ & $95(76 / 80)$ \\
\hline
\end{tabular}

MLD, DPS, DTP, PT and effective radiation dose are reported as mean. We also indicated the complications rate, including both major and minor complications, and the diagnostical success rate. All data are reported for both trial and control groups. MLD, maximum lesion diameter; DPS, distance between lesion and pleural surface; DTP, distance travelled by the needle; PT, procedure timing.

increasing the number of passages through the pleura. The advantage of coaxial technique is that the introduction and fire of the biopsy needle can be repeated many times in order to obtain adequate specimens with only one access through the pleura surface.

In our study, for each patient, a single pleural access was achieved and at least two core-biopsy specimens were obtained. Whenever the stylet of the coaxial needle was removed to replace the biopsy needle, it was filled with saline in order to reduce the risk of air embolism.

A local anaesthesia $(10-20 \mathrm{~mL}$ mepivacaine hydrochloride $2 \%$ on the parietal surface of the pleura) and a conscious mild sedation (1-2 $\mathrm{mg}$ of midazolam e.v.) were administered to the patient during procedures, to reduce patient's movement and improve procedure compliance during the biopsy, performing faster biopsies with more than one sample at the same time.

Evaluation of maximum lesion diameter (MLD), distance between lesion and pleural surface (DPS), distance travelled by the needle (DTP), procedure timing (PT), validity of histological sample and procedural complications were recorded for each patient of both groups.

The radiation dose to the patient's chest was estimated in trial and control groups by means of the total dose-length product (TDLP) and then the effective dose was obtained by applying the following formula:

$$
\text { Effective Radiation Dose }=\mathrm{TDLP} \times \mathrm{k}
$$

where $\mathrm{k}$ is the conversion factor (chest: $\mathrm{k}=0.017 \mathrm{mGy} * \mathrm{~cm}$ ) (12).

PT was calculated from the first CT scan to the final CT post-biopsy control.

We described the technical success and diagnostic success for each group. We defined technical success as a complete biopsy in which the target lesion is achieved, while diagnostic success is to refer to the achievement of a histological result.

According to Society of Interventional Radiology (SIR) standards of practice committee classification of complications by outcome, procedural complications were divided into two groups: minor and major. Haemoptysis requiring hospitalization or specific therapy, thoracostomy tube placement requiring prolonged admission, catheter exchange or pleurodesis and air embolism were recorded as major complications (13).

All other complication that did not require further treatment were considered as minor.

We performed a comparison between the two groups for each variable using the $t$-test $(\mathrm{P}<0.01)$. Then it was represented by boxplot graphs.

In addition, each group was divided into two subgroups based on lesion size, according to a cut-off of $1.5 \mathrm{~cm}$ $(<1.5 \mathrm{~cm} ; \geq 1.5 \mathrm{~cm})$. We analysed the diagnostical success rate for each subgroup.

\section{Statistical analysis}

All the statistics were elaborated using IBM SPSS Statistics for Windows, version 26 (IBM Corp., Armonk, NY, USA).

\section{Results}

Both trial and control groups had the same demographics features. Each group was made of a total of 80 patients. There were no important differences between the trial and control groups in terms of age (mean age of 68 and 70 years respectively) and gender (40 men and 40 women in both groups).

Descriptive statistical analysis of both groups is reported in Table 2.

MLD, DPS and DTP did not differ significantly between the trial and the control groups $(\mathrm{P}=0.65 ; \mathrm{P}=0,94$; $\mathrm{P}=0.06$, respectively). We reported a significantly lower $\mathrm{PT}$ in patients who had lung biopsy CT guided using SIRIO, with mean difference of 9.8 min (95\% CI: 6.825-12.775; $\mathrm{P}<0.05)$. The radiation effective dose to the patient's chest was significantly decreased in patients of the trial group, 
A

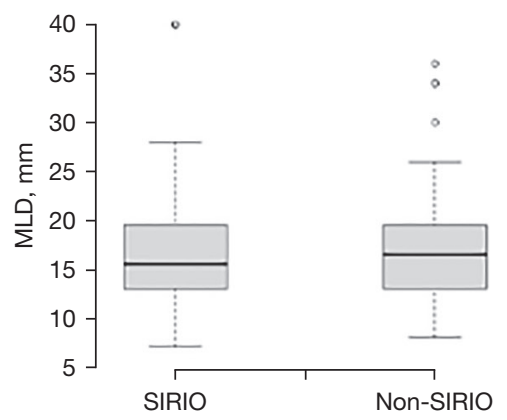

$\mathrm{D}$

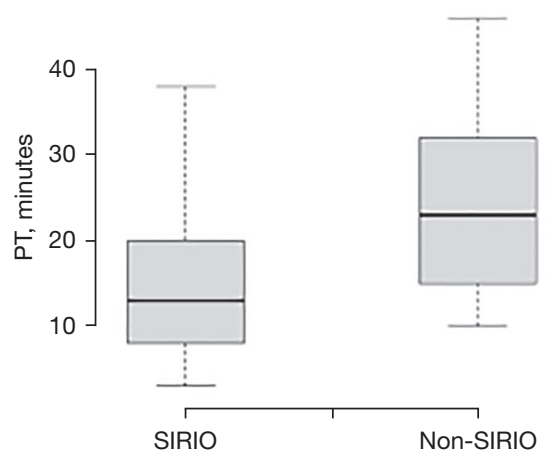

B

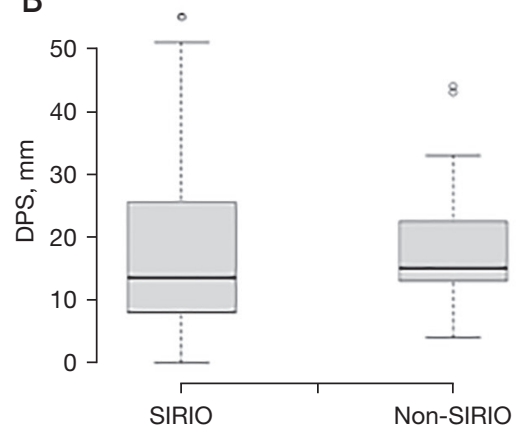

E

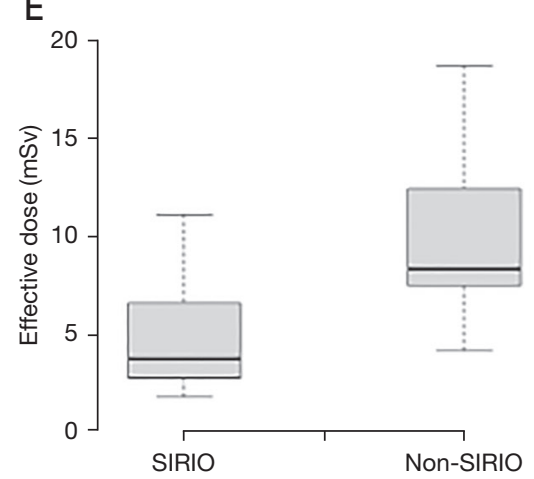

C

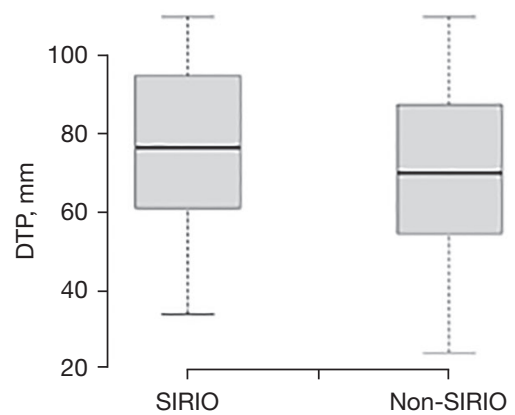

Figure 4 Boxplots show not significantly differences between the trial and the control groups for MLD (A), DPS (B) and DTP (C), while a significantly lower PT was reported in patients who had lung biopsy CT guided using SIRIO (D). In addition, we demonstrated that the radiation effective dose to the patient's chest was significantly decreased in patients of the trial group (E). MLD, maximum lesion diameter; DPS, distance between lesion and pleural surface; DTP, distance travelled by the needle; PT, procedure timing; CT, computed tomography.

with a mean difference of $5.1 \mathrm{~min}$ (95\% CI: 4.207-5.993; $\mathrm{P}<0.05)$.

Boxplots of the five variables for each group are shown in Figure 4.

Technical success was achieved in the $100 \%$ of cases in both groups. Histopathological diagnosis was obtained in $76 / 80$ cases in the trial group and in $64 / 80$ cases in the control one, so diagnostical success was higher in the trial group (95\%). Lung adenocarcinoma was the most frequent histological result with an incidence in trial and control groups of $72.5 \%$ and $68 \%$ respectively. In the trial group we did not obtain a definitive histopathological in only 4 patients, hence two of these patients were evaluated with a CT follow-up showing no progression and two patients had surgery, with a definitive histological result of adenocarcinoma. In 16 patients of the control group a definitive histopathological diagnosis was not obtained; 10 of these patients underwent a second biopsy without a definitive diagnosis so they had surgery with the result of adenocarcinoma and the other 6 patients went to CT follow-up showing no progression.

We divided each group into two subgroups based on lesion size, according to a cut-off of $1.5 \mathrm{~cm}(<1.5 \mathrm{~cm}$; $\geq 1.5 \mathrm{~cm})$. The trial group was composed by 37 patients with GGO $<1.5 \mathrm{~cm}$ and 43 with $\mathrm{GGO} \geq 1.5 \mathrm{~cm}$. In the control group there were 28 patients with GGO $<1.5 \mathrm{~cm}$ and 52 with GGO $\geq 1.5 \mathrm{~cm}$. We found that using SIRIO increases the diagnostical success rate for lesion $<1.5 \mathrm{~cm}$. We reported a diagnostical success rate of $89 \%$ and of $50 \%$ in the subgroup of patients with GGO $<1.5 \mathrm{~cm}$ in the trial and the control group, respectively, as is shown in Table 3.

Complications were recorded in $26(32.5 \%)$ and 35 (43.7\%) patients, respectively in the trial and control group, with a significant difference for major complications $(\mathrm{P}<0.05)$. No significant differences were reported for minor complications between the two groups.

In particular, in the trial group we reported only 2 cases of pneumothorax that required a thoracostomy tube positioning. The other 24 patients had minor complications; 8 patients had self-limited pneumothorax and 16 patients 
Table 3 The table describes the number of patients with a definitive histopathological diagnosis and the diagnostical success rate for both trial and control groups, according to GGO size $(<1.5 \mathrm{~cm}$ and $\geq 1.5 \mathrm{~cm})$

\begin{tabular}{lcccc}
\hline \multirow{2}{*}{ Groups } & \multicolumn{2}{c}{ Trial } & \multicolumn{2}{c}{ Control } \\
\cline { 2 - 3 } & GGO $<1.5 \mathrm{~cm}$ & GGO $\geq 1.5 \mathrm{~cm}$ & $\mathrm{GGO}<1.5 \mathrm{~cm}$ & $\mathrm{GGO} \geq 1.5 \mathrm{~cm}$ \\
\hline Patients with a definitive histopathological diagnosis & $33 / 37$ & $43 / 43$ & $14 / 28$ & $50 / 52$ \\
Diagnostical success rate (\%) & 89 & 100 & 50 & 96 \\
\hline
\end{tabular}

GGO, ground glass opacity.

self-limited haemoptysis. In the control group there were 8 pneumothorax that required chest tube drainage. The other 27 patients had minor complications. In particular, we reported 12 self-limited pneumothorax and 15 self-limited haemoptysis.

\section{Discussion}

Percutaneous image-guided biopsy is a common and well-established procedure used for the diagnosis of lung nodules. It plays a very important role in oncology and it is improving thanks to progress in knowledge of targeted therapies (14). CT is the most common imaging modality used to guide the biopsy, followed by ultrasound (US) used when lesions are next to the pleural surface (15). The patient's positioning should be chosen depending on different criteria, particularly the lesion's location and the patient's ability to tolerate positioning; when it is possible, the prone position is preferred (16). The coaxial core biopsy with an 18-gauge needle was used to reduce complications rate (17). The most common complication of PLB is pneumothorax, followed by pulmonary haemorrhage. From $1 \%$ to $14 \%$ of cases, pneumothorax can be severe and it requires placement of a drainage. Tumour seeding and air embolism are extremely rare. But the latter is a lifethreatening complication (18-21).

Few studies exist in literature about CT-guided pulmonary GGO biopsy.

Shimizu et al. (22) reported a very low diagnostic accuracy of CT-guided needle aspiration for GGO. In particular, it is reported to be lower than that for solid lesions because GGOs aspiration may result inadequate and poor in cellularity.

Zheng et al. (23) analysed 38 patients with persistent pulmonary GGO lesions $\leq 2 \mathrm{~cm}$ in size, who underwent to percutaneous CT-guided lung biopsies using an 18-gauge Monopty fully automated cutting needle. All patients received diagnostic chest-CT before the biopsy and during the procedure to plan the needle path. The incidence of cancer was about $68.4 \%$. Three $(7.9 \%)$ patients had pneumothorax and $4(10.5 \%)$ patients had mild parenchymal haemorrhage.

Lu et al. (24) performed CT-guided lung biopsy on 1,612 patients with a coaxial technique using a $19 \mathrm{G}$ guiding needle and a 20G spring-loaded semi-automatic. Only 47 patients were diagnosed with lung adenocarcinomas with a high incidence of pneumothorax (47.3\%) and of selflimited haemoptysis (20.0\%).

Unfortunately, estimated radiation dose to the patients is not comparable because it has not been evaluated in these previous studies.

A good alternative to the standard CT-guided technique is represented by CT fluoroscopy-guided biopsy. It allows a real-time visualization, but with the risk of a high amount of radiation both to patients and doctors $(25,26)$. Yamagami et al. (27) analysed 85 percutaneous lung biopsies performed under CT-fluoroscopic guidance for GGO lesions. They used both 18-gauge Monopty fully automated needle and 18-gauge Temno needle, which is a semiautomated cutting needle. A CT-fluoroscopic imaging system was used for all CT-guided biopsy procedures. Rates of adequate specimens obtained and of precise diagnosis by needle biopsy were $92.9 \%$ and $90.6 \%$ of evaluated lung lesions, respectively. The incidence of pneumothorax and haemoptysis was about $51.8 \%$ and $10.6 \%$ respectively. The mean time required for biopsy procedures was $19.7 \pm 6.4 \mathrm{~min}$. Moreover, the patient radiation dose is lower in our study than that reported in literature for PLB CT-fluoroscopy guided.

Relatively recently, Cone-Beam CT (CBCT) has been introduced. It is a system composed by an $\mathrm{X}$-ray tube, a C-arc and a flat-panel-type detector (28). Its use has increased the spatial resolution thanks to its real-time fluoroscopic and 3D abilities (29). A recent study (30) evaluated the diagnostic capacity of CBCT-guided transthoracic percutaneous biopsies on lung lesions in a group of 94 patients with 98 biopsies. They reported a technical success of about 
$96.8 \%$, similar to that we had in our study. However, a higher incidence of major complications has been demonstrated. In terms of radiation dose, the use of CBCT for PLB shows the limitation due to the significant radiation burden both to operators and patients. The effective radiation dose of the patients estimated with $\mathrm{CBCT}$ was higher than the one reported in our trial group.

According to our knowledge, there are not studies in literature about the use of an augmented reality navigation system for percutaneous CT-guided pulmonary GGO biopsies.

In previous studies we have already demonstrated that this CT-guided navigation system is safe and effective for lung biopsy, independently on lesion size and location. In addition, it reduces procedural time, pneumothorax rate and gives a better histological result in lesions $<20 \mathrm{~mm}$ compared to a standard CT-guided technique with a lower radiation exposure.

The radiation dose to the patients reported in our study is similar to that we have already demonstrated for lung biopsies of both solid and ground glass nodules (9). This is to refer to the same low dose CT-protocol we have used.

The aim of this study is to evaluate the effectiveness of this augmented reality infrared navigation system performance on CT-guided percutaneous lung GGO biopsy compared to a standard CT-guided technique.

The use of augmented reality system has shown high safety, technical reliability and effectiveness for percutaneous CT-guided pulmonary ground-glass opacity biopsies. In fact, it can give real-time information about position of anatomical structures and instruments, particularly needle progression during biopsy with a low incidence of complications and a reduction of the radiation dose administered to patients.

The great advantage of augmented reality navigation system, compared to the traditional CT-guided biopsy, is to perform a real-time procedure: the needle can be advanced through the thorax wall and the lung parenchyma using infrared (as in our system) or electromagnetic fields. The CT scan is performed only at the beginning of the procedure, when DICOM images are sent to the visualization unit, and they are not necessary to control every single needle advancement, as requested in the standard technique. This means a faster procedure, reducing CT scans acquisitions and radiation dose exposure, improving patient comfort and increasing the number of samples in the same procedural time. The result is a more safe and effective procedure than the standard CT-guided one, with advantageous benefits/costs ratio.

Thanks to procedural time reduction and patient comfort improvement, using a coaxial technique, it is possible to perform more than one sample of the same ground glass lesion in order to improve the result of histological diagnosis. The latter, in fact, may frequently result inadequate because of the low cellularity of GGO lesions.

For these reasons, a navigation system could impact the results of PLB, in particular for GGO.

The limitations of our study are that it is monocentric and retrospective. Nevertheless, it counts a high number of cases among other similar studies in the literature about pulmonary GGO biopsy. In particular, to our knowledge, there are no studies in the literature about the use of an augmented reality navigation system for GGO percutaneous CT-guided biopsies.

\section{Conclusions}

In conclusion, the use of an augmented reality navigation system for percutaneous CT-guided pulmonary GGO biopsies has demonstrated a lot of advantages compared to a standard CT-guided technique. It showed a lower incidence of post-procedural complications and a significantly reduction of the radiation dose administered to patients. We also demonstrated a higher diagnostical success rate in patients who underwent to GGO biopsies with SIRIO, particularly when GGO were $<1.5 \mathrm{~cm}$ in size.

\section{Acknowledgments}

Funding: None.

\section{Footnote}

Reporting Checklist: The authors have completed the STROBE reporting checklist. Available at https://jtd. amegroups.com/article/view/10.21037/jtd-21-1285/rc

Data Sharing Statement: Available at https://jtd.amegroups. com/article/view/10.21037/jtd-21-1285/dss

Peer Review File: Available at https://jtd.amegroups.com/ article/view/10.21037/jtd-21-1285/prf

Conflicts of Interest: All authors have completed the ICMJE uniform disclosure form (available at https://jtd.amegroups. com/article/view/10.21037/jtd-21-1285/coif). PC serves 
as an unpaid editorial board member of Fournal of Thoracic Disease from November 2016 to October 2022. The other authors have no conflicts of interest to declare.

Ethical Statement: The authors are accountable for all aspects of the work in ensuring that questions related to the accuracy or integrity of any part of the work are appropriately investigated and resolved. This study was approved by the Institutional Review Board (01/17 OSS ComEt CBM) and it was conducted in accordance with the Declaration of Helsinki (as revised in 2013). All participants gave informed consent before taking part in the study.

Open Access Statement: This is an Open Access article distributed in accordance with the Creative Commons Attribution-NonCommercial-NoDerivs 4.0 International License (CC BY-NC-ND 4.0), which permits the noncommercial replication and distribution of the article with the strict proviso that no changes or edits are made and the original work is properly cited (including links to both the formal publication through the relevant DOI and the license). See: https://creativecommons.org/licenses/by-nc-nd/4.0/.

\section{References}

1. Gao JW, Rizzo S, Ma LH, et al. Pulmonary ground-glass opacity: computed tomography features, histopathology and molecular pathology. Transl Lung Cancer Res 2017;6:68-75.

2. Kakinuma R, Muramatsu $\mathrm{Y}$, Kusumoto M, et al. Solitary Pure Ground-Glass Nodules $5 \mathrm{~mm}$ or Smaller: Frequency of Growth. Radiology 2015;276:873-82.

3. Chen D, Dai C, Kadeer X, et al. New horizons in surgical treatment of ground-glass nodules of the lung: experience and controversies. Ther Clin Risk Manag 2018;14:203-11.

4. Lim HJ, Ahn S, Lee KS, et al. Persistent pure ground-glass opacity lung nodules $\geq 10 \mathrm{~mm}$ in diameter at CT scan: histopathologic comparisons and prognostic implications. Chest 2013;144:1291-9.

5. DiBardino DM, Yarmus LB, Semaan RW. Transthoracic needle biopsy of the lung. J Thorac Dis 2015;7:S304-16.

6. Wood BJ, Zhang H, Durrani A, et al. Navigation with electromagnetic tracking for interventional radiology procedures: a feasibility study. J Vasc Interv Radiol 2005;16:493-505.

7. Meier-Meitinger M, Nagel M, Kalender W, et al. Computer-assisted navigation system for interventional CT-guided procedures: results of phantom and clinical studies. Rofo 2008;180:310-7.

8. Kelloff GJ, Sigman CC. Cancer biomarkers: selecting the right drug for the right patient. Nat Rev Drug Discov 2012;11:201-14.

9. Grasso RF, Faiella E, Luppi G, et al. Percutaneous lung biopsy: comparison between an augmented reality $\mathrm{CT}$ navigation system and standard CT-guided technique. Int J Comput Assist Radiol Surg 2013;8:837-48.

10. Grasso RF, Luppi G, Cazzato RL, et al. Percutaneous computed tomography-guided lung biopsies: preliminary results using an augmented reality navigation system. Tumori 2012;98:775-82.

11. Grasso RF, Cazzato RL, Luppi G, et al. Percutaneous lung biopsies: performance of an optical CT-based navigation system with a low-dose protocol. Eur Radiol 2013;23:3071-6.

12. Union $\mathrm{PO}$ of the $\mathrm{E}$. European guidelines on quality criteria for computed tomography 2000. Available online: http://op.europa.eu/en/publication-detail/-/publication/ d229c9e1-a967-49de-b169-59ee68605f1a

13. Gupta S, Wallace MJ, Cardella JF, et al. Quality improvement guidelines for percutaneous needle biopsy. J Vasc Interv Radiol 2010;21:969-75.

14. Rivera MP, Mehta AC, Wahidi MM. Establishing the diagnosis of lung cancer: Diagnosis and management of lung cancer, 3rd ed: American College of Chest Physicians evidence-based clinical practice guidelines. Chest 2013;143:e142S-65S.

15. Birchard KR. Transthoracic needle biopsy. Semin Intervent Radiol 2011;28:87-97.

16. Winokur RS, Pua BB, Sullivan BW, et al. Percutaneous lung biopsy: technique, efficacy, and complications. Semin Intervent Radiol 2013;30:121-7.

17. Lal H, Neyaz Z, Nath A, et al. CT-guided percutaneous biopsy of intrathoracic lesions. Korean J Radiol 2012;13:210-26.

18. Covey AM, Gandhi R, Brody LA, et al. Factors associated with pneumothorax and pneumothorax requiring treatment after percutaneous lung biopsy in 443 consecutive patients. J Vasc Interv Radiol 2004;15:479-83.

19. Wu CC, Maher MM, Shepard JA. Complications of CT-guided percutaneous needle biopsy of the chest: prevention and management. AJR Am J Roentgenol 2011;196:W678-82.

20. Khan MF, Straub R, Moghaddam SR, et al. Variables affecting the risk of pneumothorax and intrapulmonal hemorrhage in CT-guided transthoracic biopsy. Eur Radiol 2008;18:1356-63. 
21. Sabatino V, Russo U, D'Amuri F, et al. Pneumothorax and pulmonary hemorrhage after CT-guided lung biopsy: incidence, clinical significance and correlation. Radiol Med 2021;126:170-7.

22. Shimizu K, Ikeda N, Tsuboi M, et al. Percutaneous CTguided fine needle aspiration for lung cancer smaller than $2 \mathrm{~cm}$ and revealed by ground-glass opacity at CT. Lung Cancer 2006;51:173-9.

23. Zheng YF, Jiang LM, Mao WM, et al. Percutaneous computed tomography-guided lung biopsy of solitary nodular ground-glass opacity. J Cancer Res Ther 2015;11 Suppl:C231-3.

24. Lu CH, Hsiao CH, Chang YC, et al. Percutaneous computed tomography-guided coaxial core biopsy for small pulmonary lesions with ground-glass attenuation. J Thorac Oncol 2012;7:143-50.

25. Kim GR, Hur J, Lee SM, et al. CT fluoroscopy-guided lung biopsy versus conventional CT-guided lung biopsy: a prospective controlled study to assess radiation doses and

Cite this article as: Faiella E, Messina L, Castiello G, Bernetti C, Pacella G, Altomare C, Andresciani F, Sarli M, Longo F, Crucitti P, Beomonte Zobel B, Grasso RF. Augmented reality $3 \mathrm{D}$ navigation system for percutaneous CT-guided pulmonary ground-glass opacity biopsies: a comparison with the standard CT-guided technique. J Thorac Dis 2022;14(2):247-256. doi: 10.21037/jtd-21-1285 diagnostic performance. Eur Radiol 2011;21:232-9.

26. Paulo G, Bartal G, Vano E. Radiation Dose of Patients in Fluoroscopically Guided Interventions: an Update. Cardiovasc Intervent Radiol 2021;44:842-8.

27. Yamagami T, Yoshimatsu R, Miura H, et al. Diagnostic performance of percutaneous lung biopsy using automated biopsy needles under CT-fluoroscopic guidance for ground-glass opacity lesions. Br J Radiol 2013;86:20120447.

28. Wallace MJ, Kuo MD, Glaiberman C, et al. Threedimensional C-arm cone-beam CT: applications in the interventional suite. J Vasc Interv Radiol 2009;20:S523-37.

29. Jin KN, Park CM, Goo JM, et al. Initial experience of percutaneous transthoracic needle biopsy of lung nodules using C-arm cone-beam CT systems. Eur Radiol 2010;20:2108-15.

30. Gulias-Soidan D, Crus-Sanchez NM, Fraga-Manteiga D, et al. Cone-Beam CT-Guided Lung Biopsies: Results in 94 Patients. Diagnostics (Basel) 2020;10:1068. 